\title{
Vitamin D supplementation in patients with type 2 diabetes
}

\author{
Dharshini Karuppiah ${ }^{1}$, Sivatharshya Pathmanathan ${ }^{2}$ \\ ${ }^{1}$ Diabetes and Endocrinology unit, Teaching Hospital Batticaloa \\ ${ }^{2}$ Diabete and Endocrinology unit, District General Hospital Kalutara
}

Vitamin D, the so called sun-shine vitamin, is a hot topic currently, with number of studies proclaiming its benefits for several non-skeletal illnesses including cancer, cardiovascular diseases, autoimmune and inflammatory diseases, dementia, and diabetes. Conversely, recent studies have been more cautious and questioning its perceived benefits (1).

Along with numerous non-skeletal effects, vitamin D has received its attention on potential pancreatic beta cell function and insulin sensitivity. Historically the role of vitamin $\mathrm{D}$ in diabetes was recognized by a seasonal variation in glycemic control reported in patients with diabetes being worse in the winter. This may be due to insufficient vitamin D as a result of reduced sunlight in winter. Additional evidence supported the role of vitamin $\mathrm{D}$ in diabetes; presence of vitamin $\mathrm{D}$ receptors on pancreatic $\beta$ cells, expression of $1 \alpha$ hydroxylase activity at pancreatic $\beta$ cells causing activation of vitamin $D$, and increased transcription of insulin receptor genes by $1,25(\mathrm{OH}) \mathrm{D}$ (2). As $\beta$-cells in the pancreas are affected via cytokine-induced apoptosis, systemic inflammation has an important role in insulin resistance and cardiovascular events in patients with diabetes. Vitamin D could decrease the effects of systemic inflammation and protect $\beta$-cells. Other proposed mechanism where vitamin $\mathrm{D}$ affects insulin resistance is through the renin angiotensin aldosterone system (RAAS). Low levels of $1,25(\mathrm{OH}) 2 \mathrm{D}$ is shown to increase angiotensin II which inhibit the action of insulin in vascular and skeletal muscle tissues, leading to impaired glucose uptake (3).

Large number of cross-sectional studies have generally reported an inverse association between vitamin D status and prevalent hyperglycemia, favouring the biological perspective, where vitamin $\mathrm{D}$ have both direct and indirect effects on various mechanisms related to the pathophysiology of type 2 diabetes.

The study by Forouhi et al. reported that the baseline serum vitamin $\mathrm{D}$ levels are inversely associated with glucose and insulin levels collected 10 years later(4). Finnish cohort study also showed an inverse association between baseline serum 25(OH) D and 17-year risk of type 2 diabetes, after adjustment for confounders like obesity and low physical activity (5). A post hoc analysis of a trial designed for bone-related outcomes, found that
$700 \mathrm{IU} /$ day of Vitamin D3 (combined with calcium) decreased homeostasis model assessment of insulin resistance in participants with impaired glucose tolerance but not in those with normal fasting glucose. Numerous other prospective cohort studies have also demonstrated that higher vitamin D status was associated with reduced risk of type 2 diabetes. The important role of vitamin D in the development of diabetes evidenced by these epidemiological studies prompts clinicians to prescribe more and more vitamin D supplements. Subsequently several researches on the positive preventive effect of vitamin D supplementation were widely carried out. Number of randomized controlled trials study showed that vitamin D supplementation reduced blood glucose, increased insulin sensitivity in diabetes patients, and decreased development of diabetes related complications (6).

On the other hand, there was a lack of correlation between the use of vitamin $\mathrm{D}$ and insulin secretion rate or hemoglobin A1c (HbA1c) in patients with diabetes (7). Findings from the investigation by Dr Forouhi and colleagues using data from several studies covering thousands of people of European descent also supported the same. They investigated the link between levels of Vitamin D and risk of developing diabetes by examining the genes that control blood levels of Vitamin D. The researchers did not find any evidence of low Vitamin D causing type 2 diabetes, nor they found a link between the risk of developing type 2 diabetes and the different gene variants that control blood levels of Vitamin $\mathrm{D}$ (2).

More recently the findings from the Vitamin D and Type 2 Diabetes (D2d) trial were presented at the American Diabetes Association 2019 scientific sessions by Anastassios Pittas. Among persons at high risk for type 2 diabetes not selected for vitamin $\mathrm{D}$ insufficiency, vitamin D3 supplementation at a dose of $4000 \mathrm{IU}$ per day did not result in a significantly lower risk of diabetes than placebo. Study results did not show a statistically significant benefit for vitamin $\mathrm{D}$ in decreasing progression to type 2 diabetes in people who have sufficient levels. However, in a post-hoc analysis of data from the 103 participants with vitamin D deficiency (< $12 \mathrm{ng} / \mathrm{mL}$ ) showed that vitamin $\mathrm{D}$ supplementation potentially had a benefit (7). 
Other recent systemic analysis also suggested that large dosage, short-term vitamin D supplementation was most likely to yield preferred changes in vitamin $\mathrm{D}$ deficient, non-obese groups, Asians, especially Middle Easterners, and patients with optimal glycemic control at baseline (8)

Even though Sri Lanka is a tropical country with sufficient sunlight throughout the year, vitamin D insufficiency is not uncommon among our population. But data on vitamin D status in Sri Lankans are very few. One study showed that vitamin D status among Sri Lankans living in Kandy was reasonable levels (mean 54.2 $\mathrm{nmol} / \mathrm{l})$ and a clear seasonal variation with the lowest s25(OH)D levels in August-September and the highest levels in November-December $\left.{ }^{(9}\right)$. In the national survey, a higher prevalence of calcium deficiency was found among children aged 6-59 months. But there was a marked variation in the prevalence of hypovitaminosis D from 5 to $25 \%$ (10). A recent study done in an urban setting in Colombo, Sri Lanka showed $90.2 \%$ of cumulative community prevalence of vitamin $\mathrm{D}$ deficiency and insufficiency. Prevalence was highest among young and females. Moor ethnicity showed a significant association with vitamin $\mathrm{D}$ deficiency.
Dysglycaemia was highly prevalent in this population, but it was not associated with vitamin D deficiency(11).

In conclusion, there is a biological plausibility of an important role of vitamin $\mathrm{D}$ in type 2 diabetes, and lower vitamin $\mathrm{D}$ status and intake are associated with higher risk of incident type 2 diabetes in observational studies; however, the effect of vitamin D supplementation on glycaemic outcomes was inconsistent. Currently there is insufficient data to support the contention that type 2 diabetes can be improved by raising vitamin D concentration. In Sri Lanka confirmation of a potential beneficial effect of vitamin $\mathrm{D}$ on type 2 diabetes in our population need to studied in large trials. This should be specifically designed to test the vitamin $\mathrm{D}$ status in our population and whether this is a direct contributor to type 2 diabetes pathogenesis. If such an intervention proven effective, this measures could be applied at public health level to decrease diabetes related burden and costs.

\section{References}

1. JoAnn E. Manson, M.D., et al. Vitamin D Supplements and Prevention of Cancer and Cardiovascular Disease. N Engl J Med 2019;380:33-44 DOI: 10.1056/NEJMoa1809944

2. Acharya A and Halemani SS: Role of Vitamin D in Diabetes Mellitus. Int J Pharm Sci Res 2016; 7(5): 1881-88.doi: 10.13040/IJPSR.0975-8232.7(5).1881-88.

3. Nakashima, Akio et al. "Role of vitamin D in diabetes mellitus and chronic kidney disease." World journal of diabetes vol. 7,5 (2016): 89-100. doi:10.4239/wjd.v7.i5.89.

4. Forouhi NG, Luan J, Cooper A, Boucher BJ, Wareham N. Baseline 25-hydroxy vitamin D is predictive of future glycemic status and insulin resistance: the Medical Research Council Ely prospective study. Diabetes2008; 57:2619- 25.

5. Mattila C, Knekt P, Mannisto S, Rissanen H, Laaksonen MA, Montonen J, Reunanen A: Serum 25-hydroxyvitamin D concentration and subsequent risk of type 2 diabetes. Diabetes Care 2007;30:2569-70.

6. Rad EY, Djalali M, Koohdani F, et al. The effects of vitamin D supplementation on glucose control and insulin resistance in patients with diabetes type 2: a randomized clinical trial study. Iranian J Public Health 2014;43:1651-6.

7. Pittas AG, Harris SS, Stark PC, Dawson-Hughes B. The effects of calcium and vitamin D supplementation on blood glucose and markers of inflammation in nondiabetic adults. Diabetes Care 2007;30:980- 6.

8. Xinyi Li ID, Yan Liu, Yingdong Zheng, Peiyu Wang and Yumei Zhang. The Effect of Vitamin D Supplementation on Glycemic Control in Type 2 Diabetes Patients: A Systematic Review and Meta-Analysis. Nutrients 2018, 10, 375; doi:10.3390/nu10030375

9. Haakon E Meyer et.al. Vitamin D status in Sri Lankans living in Sri Lanka and Norway. British Journal Of Nutrition 99(5):941$4 \cdot$ May 2008

10. Abeywickrama HM, Koyama Y, Uchiyama M, et al. Micronutrient Status in Sri Lanka: A Review. Nutrients. 2018;10(11):1583. Published 2018 Oct 27. doi:10.3390/nu10111583

11. Chandrika J Subasinghe. Et al. Prevalence of vitamin D deficiency/Insufficiency and its metabolic associations in an urban setting in Sri Lanka: Data from Colombo Urban study. SJDEM. 2019;9(2) 